\title{
Long range surface polaritons supported by lossy thin films
}

\author{
Christophe Arnold, ${ }^{\text {a) }}$ Yichen Zhang, and Jaime Gómez Rivas \\ Center for Nanophotonics, FOM Institute AMOLF, clo Philips Research Laboratories, \\ High Tech Campus 4, 5656 AE Eindhoven, The Netherlands
}

(Received 22 December 2009; accepted 24 February 2010; published online 19 March 2010)

\begin{abstract}
We demonstrate experimentally that strongly absorbing chalcogenide thin films can support long range surface polaritons. Moreover, the possibility to change the phase of this material allows us to investigate the influence of the permittivity of the thin film on the surface modes. We demonstrate the relative insensitivity of these modes to the permittivity of the thin film. Extending the range of materials that support long range surface polaritons to strongly absorbing layers opens new possibilities for applications that were thought to be limited to weakly absorbing media. (C) 2010 American Institute of Physics. [doi:10.1063/1.3364938]
\end{abstract}

Surface polaritons are electromagnetic waves propagating at the interface between two media. The electromagnetic field of surface polaritons decays exponentially with distance from the interface. When a thin film is embedded in between two similar dielectrics, two surface polaritons can be excited on both interfaces of the film. These surface polaritons are coupled, and their degeneracy is lifted if the film thickness is small enough. These surface waves have been intensively investigated in thin films of noble metals, such as gold and silver, ${ }^{1-5}$ and several applications have been proposed. ${ }^{6}$ Extending the range of materials used to support surface waves could lead to a broaden spectrum of applications, such as sensors and waveguides. In this article, we study the excitation of surface polariton modes in thin films of strongly absorbing materials, demonstrating that these modes can be supported independently of the value of the real part of the permittivity of the thin film.

Only modes with a wave number inside the light cone are supported at the interface between a nonlossy and a lossy dielectric. However, the coupling of these modes at the opposite sides of a thin film of lossy dielectric surrounded by a nonlossy dielectric gives rise to surface modes with a dispersion relation outside the light cone. These modes, which can propagate a long distance along the thin film, are called long range surface polaritons (LRSPs). ${ }^{7-9}$ There are only a few works on these LRSPs, most probably because of the uncommon conditions for their existence: $\left|\epsilon_{r}\right| \lesssim \epsilon_{i}$ and $\epsilon_{i} \gg 1$, being $\epsilon_{r}$ and $\epsilon_{i}$ the real and imaginary components of the permittivity of the thin film. Kovacs ${ }^{7}$ demonstrated that LRSPs can be excited onto an iron thin film by the attenuated total reflection method. Yang et al. ${ }^{9,10}$ showed that a thin film of Vanadium can support surface waves in the infrared. More recently, Giannini et al. ${ }^{11}$ have demonstrated that LRSPs can be excited on a silicon thin film in the UV-visible range. Here, we investigate LRSPs supported by chalcogenide $\mathrm{Ge}_{17} \mathrm{Sb}_{76} \mathrm{Te}_{7}$ (GST) thin films at visible frequencies. The phase of this material can be changed between a crystalline (c-GST) and amorphous (a-GST) phase by varying temperature. A phase change also strongly modifies the permittivity of the thin film. This property is the reason why chalcogenide materials are commonly used in optical memory devices and electronic solid state devices. ${ }^{12}$ In Ref. 9, it was pre-

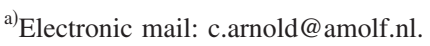

dicted that LRSPs supported by very lossy materials are insensitive to changes in the real part of the permittivity. By taking advantage of the different phases in GST, we demonstrate this prediction.

The investigated sample is composed of a substrate of Schott F2 glass with a permittivity $\epsilon=2.62$ at $\lambda=600 \mathrm{~nm}$. On top of the substrate, we have deposited by electron beam sputtering a $370 \mathrm{~nm}$ layer of silica and a $19 \mathrm{~nm}$ layer of GST. The thickness of the silica layer has been determined from a side-view scanning electron microscope image of the cleaved sample made after the optical measurements. The thickness of the chalcogenide thin layer in its amorphous phase has been obtained by x-ray reflectometry. The roughness of the two interfaces of the GST thin film, estimated from the X-ray reflectometry, is $4 \mathrm{~nm}$. We used a liquid (Cargille Laboratories) to match the refractive index of the $\mathrm{SiO}_{2}$ top layer in order to have a symmetric medium surrounding the thin film. Refractive indices of the matching index liquid and of the silica layer have been determined by ellipsometry measurements. The difference between the two indices is less than 0.01 in the range of investigated wavelengths. The attenuated total reflection (ATR) method ${ }^{1}$ was used to excite the surface mode (see Fig. 1). In this method, a prism of F2 glass, index matched to the sample substrate, is used to illuminate the $\mathrm{F} 2-\mathrm{SiO}_{2}$ interface at an angle larger than the critical angle for total internal reflection. The evanescent transmitted amplitude can couple to LRSPs at the resonant wavelengths and angles, leading to a reduction in the reflection. We measured

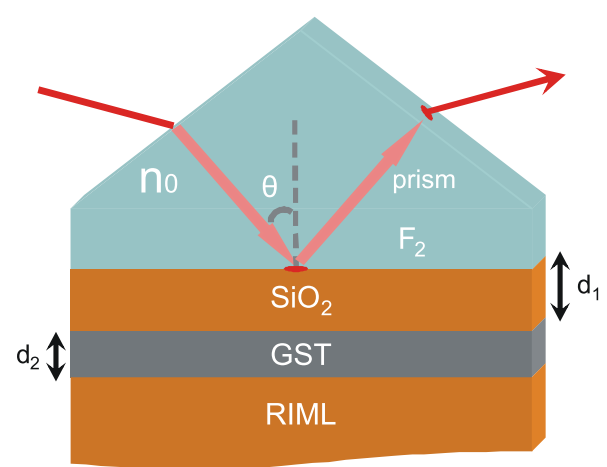

FIG. 1. (Color online) Schematic representation of the sample and the experimental method. $d_{1}$ is the thickness of the upper $\mathrm{SiO}_{2}$ layer, $d_{2}$ is the thickness of the thin GST layer. The refractive index matching liquid is sufficiently thick to be considered infinite. 
(a)

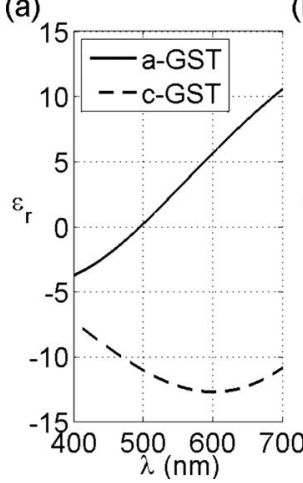

(b)

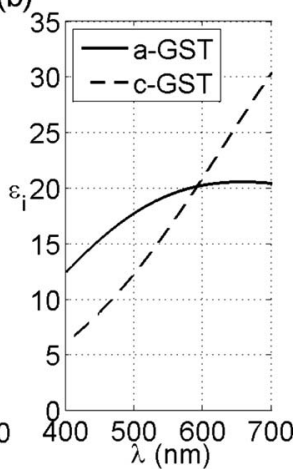

FIG. 2. Permittivity of GST. The solid lines correspond to the amorphous phase and the dashed lines to the crystalline phase. Figure (a) displays the real component of the permittivity, while (b) shows the imaginary component.

the specular reflection on the $\mathrm{F} 2-\mathrm{SiO}_{2}$ interface by varying the angle of incidence of a beam at optical frequencies. The experiments were done with a computer controlled rotation stage set-up that allowed the simultaneous rotation of sample and detector. The light source was a collimated beam from a halogen lamp (Yokogawa AQ4303), and we used a fiber coupled spectrometer (Ocean Optics USB2000) to measure the reflection. First, we measured the reflection on the sample having GST film in its amorphous phase. Then, the sample was heated up at $250{ }^{\circ} \mathrm{C}$ during $30 \mathrm{~min}$ in order to change the GST layer in its crystalline phase. Finally, we reiterated the reflection measurements on the sample having the GST thin film in its crystalline phase. The references for these measurements were obtained by measuring the reflection for each phase of the GST thin film of s-polarized light incident at an angle larger than the critical angle, i.e., reflectance close to 1 .

Figure 2 displays the permittivity of the crystalline (c-GST) and the amorphous (a-GST) chalcogenide thin film, which have been determined by ellipsometry measurements. The imaginary component [Fig. 2(b)] is large for the two phases and has a similar value around $600 \mathrm{~nm}$. The largest change between the two phases concerns the real part of the permittivity [Fig. 2(a)]: whereas c-GST has a metallic character $\left(\epsilon_{r}<0\right)$, a-GST is an absorbing dielectric above $500 \mathrm{~nm}$ $\left(\epsilon_{r}>0\right)$.

Figure 3(a) shows the reflectance of p-polarized light measured on the a-GST sample. A reduction in the total internal reflection in the prism is clearly visible near $65^{\circ}$. This resonance appears after the critical angle, which is around $63.7^{\circ}$. The minimum of reflection is almost zero (5\%), revealing the very efficient coupling of the incident light to LRSPs. Figure 3(b) displays the reflectance of the c-GST sample. The resonance is similar for the two phases of GST in terms of linewidth, resonance angle and coupling efficiency. This is better seen in Fig. 4, which displays the reflectance as a function of the angle at $\lambda=600 \mathrm{~nm}$ for both phases. The linewidths of these resonances are $1.3^{\circ}$ for c-GST and $1.7^{\circ}$ for a-GST. The difference on the resonance angle between the two phases is $0.2^{\circ}$. The difference in the reflectance at smaller angles than the critical angle confirms the different permittivity between the two phases of the thin film. The curves with symbols in Fig. 4 are fits to the measurements for the two phases calculated by solving the Fresnel's equations for the multilayer structure. The only ad-

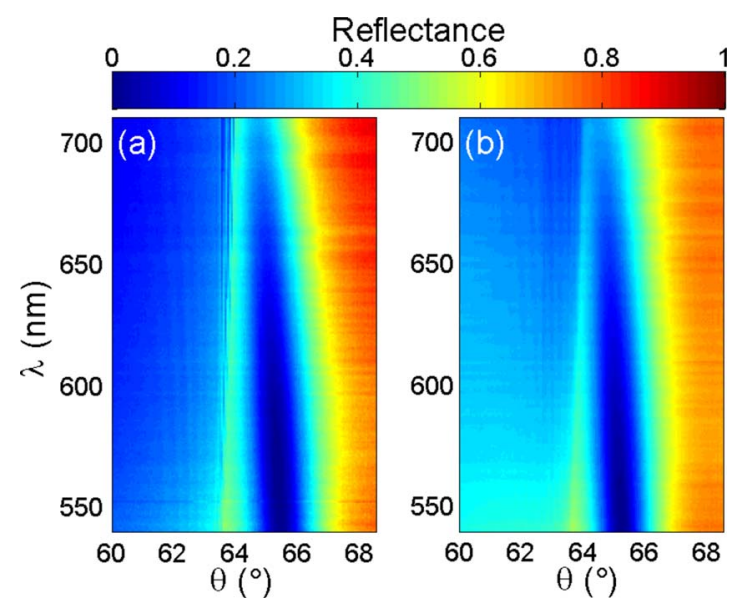

FIG. 3. (Color online) Reflectance measured in the ATR configuration as a function of the wavelength and the incident angle on amorphous-GST (a) and crystalline-GST (b) thin films.

justed parameter in the fits is the thickness of the thin film. The permittivities of each layer of the sample were fixed to the values obtained from ellipsometry measurements. From the fits, we obtain an amorphous layer with a thickness of $22.5 \pm 0.5 \mathrm{~nm}$ and a crystalline layer of $20.5 \pm 0.5 \mathrm{~nm}$. It is reasonable to think that the change in phase can slightly modify the thickness of the layer, as it has already been reported on different phase change materials. ${ }^{13}$ The small shift of the resonance is thus partly due to this variation in thickness. The thickness of the amorphous GST thin film that we obtained from the fits is in reasonable agreement with the value of $19 \mathrm{~nm}$ obtained by $\mathrm{x}$-ray reflectometry. The origin of the small discrepancy of $3.5 \mathrm{~nm}$ between both measurements is unclear. Figure 5 shows the dispersion relation of the LRSPs, i.e., $\omega=2 \pi c / \lambda$ versus $k_{\|}=2 \pi n_{\mathrm{SiO}_{2}} \sin \theta / \lambda$, for both phases. These dispersion relations have been obtained from the measurements of Fig. 3, from the minima in reflectance at each wavelength. The dot-dashed line is the light cone in silica. This figure reveals the similarity of the modes despite the large difference in the permittivity of the thin film.

To go further in the analysis of guided modes supported by thin layers, we have calculated the effective index and propagation length in the three layer system silica/thin film/ silica. Figure 6(a) represents the effective index at $\lambda$ $=600 \mathrm{~nm}$ of p-polarized modes for a film with a thickness of $20 \mathrm{~nm}$ as a function of its permittivity. These results have

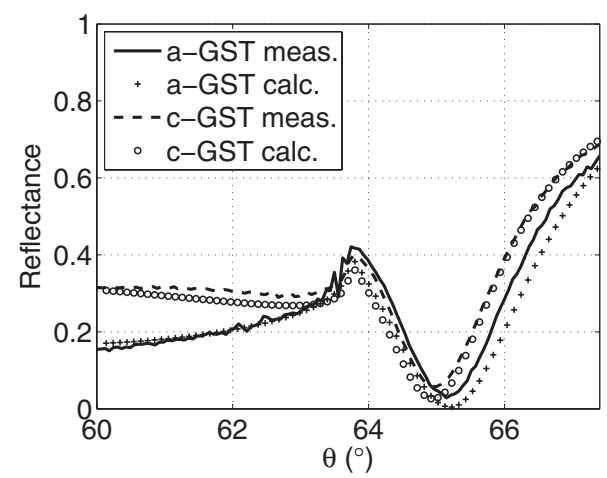

FIG. 4. Reflectance at $600 \mathrm{~nm}$ as a function of the internal angle of incidence, for the amorphous (solid curve) and crystalline (dashed curve) phases of the GST thin film. The cross and open circles are the calculated reflectances for the amorphous and crystalline thin films, respectively. 


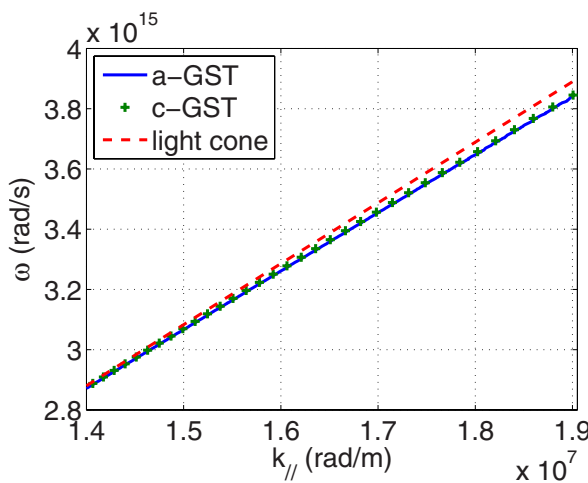

FIG. 5. (Color online) Dispersion relation of LRSPs measured for the amorphous (solid blue curve) and crystalline (green crosses) phases of the GST thin film. The red dashed line is the light cone in silica.

been obtained by looking for the poles of the reflection coefficient on this system. The $x$-axis defines the real component of the permittivity of the thin film $\epsilon_{r}$, whereas the $y$-axis is the imaginary component $\epsilon_{i}$. We can first notice that almost any material can support surface waves in this configuration. Only a small range of permittivities defined by the following relation $\epsilon_{r}^{2}+\epsilon_{i}^{2}<\epsilon_{\mathrm{SiO}_{2}} \cdot \epsilon_{r}$ and marked with the white area close to the origin on Fig. 6 do not support any surface mode. ${ }^{9}$ The square and circle in Fig. 6 represent the a-GST and c-GST thin films at $\lambda=600 \mathrm{~nm}$.

When $\epsilon_{r}^{2}+\epsilon_{i}^{2} \gg \epsilon_{\mathrm{SiO}_{2}}^{2}$, the wave vector of the mode $k=k_{r}$ $+i k_{i}$ is reduced to the expression

$$
k_{r} \simeq k_{0} \sqrt{\epsilon_{\mathrm{SiO}_{2}}}\left[1+\frac{\epsilon_{\mathrm{SiO}_{2}}}{2}\left(\frac{\pi \mathrm{d}_{2}}{\lambda}\right)^{2}\right], \quad k_{i} \simeq k_{0} \epsilon_{\mathrm{SiO}_{2}}^{3 / 2} \frac{\epsilon_{i}}{\epsilon_{r}^{2}+\epsilon_{i}^{2}},
$$

where $k_{0}$ is the vacuum wave vector and $\mathrm{d}_{2}$ is the thickness of the thin film. This expression is only valid in the thin film limit, i.e., when $\alpha=\left|\frac{1}{2} k_{\perp, t f} \cdot \mathrm{d}_{2}\right| \ll 1$, being $k_{\perp, t f}$ the normal component of the wave vector inside the thin layer. ${ }^{9}$

We can notice that the effective index of the modes, $n_{r}$ $=k_{r} / k_{0}$, becomes independent of the permittivity of the thin film when this is large enough. We can clearly see this trend in the exact calculation of Fig. 6 for a wide range of permittivities, where the value of the effective index is around 1.477, which is the value that we obtain with Eq. (1). We point out that the permittivity of the GST material is in the range of values for which the effective index of LRSPs is
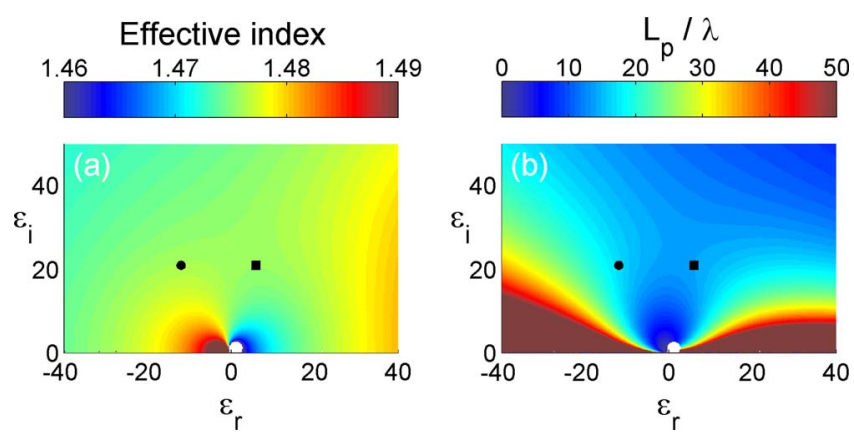

FIG. 6. (Color online) (a) Effective index as a function of the permittivity of the thin film, calculated at $\lambda=600 \mathrm{~nm}$, for a thickness of the thin film equal to $20 \mathrm{~nm}$. The symmetric surrounding media is in silica. (b) Propagation length of the modes normalized to the wavelength. The square (resp. circular) marker gives the position of the permittivity of the amorphous (resp. crystalline) GST. nearly unaffected by changes on this permittivity, which is in agreement with the measurements. Moreover, in the case of very lossy thin film, i.e., when $\epsilon_{i}^{2} \gg \epsilon_{r}^{2}, k_{i}$ in Eq. (1) reduces to $k_{0} \epsilon_{\mathrm{SiO}_{2}}^{3 / 2} / \epsilon_{i}$. In this case, neither the real part, nor the imaginary part of the wave vector of the surface polaritons depends on $\epsilon_{r}$. We can also notice that $k_{i}$ is inversely proportional to $\epsilon_{i}$, so the propagation length of the surface mode, given by $L_{p}=1 /\left(2 k_{i}\right)$, increases as the loss in the thin film increases. ${ }^{8}$ Figure $6(\mathrm{~b})$ represents the exact calculation of $L_{p}$ normalized to the wavelength, for the same range of permittivities as in Fig. 6(a). We can indeed see that the propagation length increases with $\epsilon_{i}$, when $\epsilon_{r} \ll \epsilon_{i} \leqslant 20$. However, between $\epsilon_{i} \sim 20$ and 30, the propagation length seems to be nearly constant and for $\epsilon_{i}>30, L_{p}$ decreases as $\epsilon_{i}$ increases. This discrepancy with the analytic expression of Eq. (6) is due to the fact that we are not in the thin-film limit approximation anymore. For instance, for $\epsilon=20 i$, the value of the parameter $\alpha$ is equal to 0.5 .

It is worth noticing that for a thickness of $20 \mathrm{~nm}$, both the effective index and the propagation length of LRSPs are relatively insensitive to the permittivity of the thin film on a wide range of permittivities. We emphasize that this situation can be found at any wavelength by adjusting the thickness of the thin film.

In conclusion, we have shown that a $20 \mathrm{~nm}$ thin film made of chalcogenide GST material can support LRSPs in the visible. By changing the phase of this material, we have also investigated the dependence of surface modes supported by lossy thin films with their permittivity. We have demonstrated the relative insensitivity of the mode with the real component of the permittivity of the thin film, even if the thin-film approximation is not fulfilled.

We are thankful to J. H. J. Roosen and A. P. M. de Win for technical assistance during sample preparation and characterization, H. J. Wondergem for x-ray reflectometry measurements and M. Vervest for the SEM image. We also thank V. Giannini for stimulating discussions. This work was supported by the Netherlands Foundation Fundamenteel Onderzoek der Materie (FOM) and the Nederlandse Organisatie voor Wetenschappelijk Onderzoek (NWO), and is part of an industrial partnership program between Philips and FOM.

${ }^{1}$ H. Raether, Surface Plasmons on Smooth and Rough Surfaces and on Gratings, Springer Tracts in Modern Physics (Springer, Berlin, Heidelberg, 1988), Vol. 111.

${ }^{2}$ E. N. Economou, Phys. Rev. 182, 539 (1969).

${ }^{3}$ D. Sarid, Phys. Rev. Lett. 47, 1927 (1981).

${ }^{4}$ R. Charbonneau, P. Berini, E. Berolo, and E. Lisicka-Shrzek, Opt. Lett. 25, 844 (2000).

${ }^{5}$ K. Leosson, T. Nikolajsen, A. Boltasseva, and S. I. Bozhevolnyi, Opt. Express 14, 314 (2006).

${ }^{6}$ P. Berini, Adv. Opt. Photon. 1, 484 (2009).

${ }^{7}$ G. J. Kovacs, J. Opt. Soc. Am. 68, 1325 (1978).

${ }^{8}$ F. Yang, J. R. Sambles, and G. W. Bradberry, Phys. Rev. Lett. 64, 559 (1990).

${ }^{9}$ F. Yang, J. R. Sambles, and G. W. Bradberry, Phys. Rev. B 44, 5855 (1991).

${ }^{10}$ F. Yang, G. W. Bradberry, and J. R. Sambles, J. Mod. Phys. 37, 1545 (1990).

${ }^{11}$ V. Giannini, Y. Zhang, M. Forcales, and J. G. Rivas, Opt. Express 16, 19674 (2008).

${ }^{12}$ A. Pirovano, A. Lacaita, F. Pellizzer, S. Kostylev, A. Benvenutti, and R. Bez, IEEE Trans. Electron Devices 51, 714 (2004).

${ }^{13}$ K. Shportko, S. Kremers, M. Woda, D. Lencer, J. Robertson, and M. Wuttig, Nature Mater. 7, 653 (2008) 